\title{
Gradient Estimates of Solutions to the Conductivity Problem with Flatter Insulators
}

\author{
YanYan Li and Zhuolun Yang*
}

Department of Mathematics, Rutgers University, 110 Frelinghuysen Rd, Piscataway, NJ 08854, USA

Received 7 February 2021; Accepted (in revised version) 15 February 2021

Dedicated to Prof. Paul H. Rabinowitz with admiration on the occasion of his 80th birthday

\begin{abstract}
We study the insulated conductivity problem with inclusions embedded in a bounded domain in $\mathbb{R}^{n}$. When the distance of inclusions, denoted by $\varepsilon$, goes to 0 , the gradient of solutions may blow up. When two inclusions are strictly convex, it was known that an upper bound of the blow-up rate is of order $\varepsilon^{-1 / 2}$ for $n=2$, and is of order $\varepsilon^{-1 / 2+\beta}$ for some $\beta>0$ when dimension $n \geq 3$. In this paper, we generalize the above results for insulators with flatter boundaries near touching points.
\end{abstract}

Key Words: Conductivity problem, harmonic functions, maximum principle, gradient estimates. AMS Subject Classifications: 35B44, 35J25, 35J57, 74B05, 74G70, 78A48

\section{Introduction and main results}

Let $\Omega$ be a bounded domain in $\mathbb{R}^{n}$ with $C^{2}$ boundary, and let $D_{1}^{*}$ and $D_{2}^{*}$ be two open sets whose closure belongs to $\Omega$, touching only at the origin with the inner normal vector of $\partial D_{1}^{*}$ pointing in the positive $x_{n}$-direction. Denote $x=\left(x^{\prime}, x_{n}\right)$. Translating $D_{1}^{*}$ and $D_{2}^{*}$ by $\frac{\varepsilon}{2}$ along $x_{n}$-axis, we obtain

$$
D_{1}^{\varepsilon}:=D_{1}^{*}+\left(0^{\prime}, \varepsilon / 2\right) \quad \text { and } \quad D_{2}^{\varepsilon}:=D_{2}^{*}-\left(0^{\prime}, \varepsilon / 2\right) .
$$

When there is no confusion, we drop the superscripts $\varepsilon$ and denote $D_{1}:=D_{1}^{\varepsilon}$ and $D_{2}:=$ $D_{2}^{\varepsilon}$. Denote $\widetilde{\Omega}:=\Omega \backslash \overline{\left(D_{1} \cup D_{2}\right)}$. A simple model for electric conduction can be formulated as the following elliptic equation:

$$
\begin{cases}\operatorname{div}\left(a_{k}(x) \nabla u_{k}\right)=0 & \text { in } \Omega, \\ u_{k}=\varphi(x) & \text { on } \partial \Omega\end{cases}
$$

*Corresponding author. Email addresses: yyli@math.rutgers.edu (Y. Y. Li), zy110@math.rutgers.edu (Z. Yang) 
where $\varphi \in C^{2}(\partial \Omega)$ is given, and

$$
a_{k}(x)= \begin{cases}k \in(0, \infty) & \text { in } D_{1} \cup D_{2}, \\ 1 & \text { in } \widetilde{\Omega},\end{cases}
$$

refers to conductivities. The solution $u_{k}$ and its gradient $\nabla u_{k}$ represent the voltage potential and the electric fields respectively. From an engineering point of view, It is an interesting problem to capture the behavior of $\nabla u_{k}$. Babuška, et al. [3] numerically analyzed that the gradient of solutions to an analogous elliptic system stays bounded regardless of $\varepsilon$, the distance between the inclusions. Bonnetier and Vogelius [5] proved that for a fixed $k,\left|\nabla u_{k}\right|$ is bounded for touching disks $D_{1}$ and $D_{2}$ in dimension $n=2$. A general result was obtained by Li and Vogelius [11] for general second order elliptic equations of divergence form with piecewise Hölder coefficients and general shape of inclusions $D_{1}$ and $D_{2}$ in any dimension. When $k$ is bounded away from 0 and $\infty$, they established a $W^{1, \infty}$ bound of $u_{k}$ in $\Omega$, and a $C^{1, \alpha}$ bound in each region that do not depend on $\varepsilon$. This result was further extended by Li and Nirenberg [10] to general second order elliptic systems of divergence form. Some higher order estimates with explicit dependence on $r_{1}, r_{2}, k$ and $\varepsilon$ were obtained by Dong and Li [7] for two circular inclusions of radius $r_{1}$ and $r_{2}$ respectively in dimension $n=2$. There are still some related open problems on general elliptic equations and systems. We refer to p. 94 of [11] and p. 894 of [10].

When the inclusions are insulators $(k=0)$, it was shown in $[6,9,13]$ that the gradient of solutions generally becomes unbounded, as $\varepsilon \rightarrow 0$. It was known that (see e.g., Appendix of [4]) when $k \rightarrow 0, u_{k}$ converges to the solution of the following insulated conductivity problem:

$$
\begin{cases}-\Delta u=0 & \text { in } \widetilde{\Omega}, \\ \frac{\partial u}{\partial v}=0 & \text { on } \partial D_{i}, \quad i=1,2 \\ u=\varphi & \text { on } \partial \Omega .\end{cases}
$$

Here $v$ denotes the inward unit normal vectors on $\partial D_{i}, i=1,2$.

The behavior of the gradient in terms of $\varepsilon$ has been studied by Ammari et al. in [1] and [2], where they considered the insulated problem on the whole Euclidean space:

$$
\begin{cases}\Delta u=0 & \text { in } \mathbb{R}^{n} \backslash \overline{\left(D_{1} \cup D_{2}\right)}, \\ \frac{\partial u}{\partial v}=0 & \text { on } \partial D_{i}, \quad i=1,2 \\ u(x)-H(x)=\mathcal{O}\left(|x|^{n-1}\right) & \text { as }|x| \rightarrow \infty\end{cases}
$$

They established when dimension $n=2, D_{1}^{*}$ and $D_{2}^{*}$ are disks of radius $r_{1}$ and $r_{2}$ respectively, and $H$ is a harmonic function in $\mathbb{R}^{2}$, the solution $u$ of (1.3) satisfies

$$
\|\nabla u\|_{L^{\infty}\left(B_{4}\right)} \leq C \varepsilon^{-1 / 2},
$$


for some positive constant $C$ independent of $\varepsilon$. They also showed that the upper bounds are optimal in the sense that for appropriate $H$,

$$
\|\nabla u\|_{L^{\infty}\left(B_{4}\right)} \geq \varepsilon^{-1 / 2} / C .
$$

In fact, the equation

$$
\begin{cases}\operatorname{div}\left(a_{k}(x) \nabla u_{k}\right)=0 & \text { in } \mathbb{R}^{2} \backslash \overline{\left(D_{1} \cup D_{2}\right)}, \\ u(x)-H(x)=\mathcal{O}\left(|x|^{-1}\right) & \text { as }|x| \rightarrow \infty\end{cases}
$$

was studied there, and the estimates derived have explicit dependence on $r_{1}, r_{2}, k$ and $\varepsilon$.

Yun extended in [14] and [15] these results allowing $D_{1}^{*}$ and $D_{2}^{*}$ to be any bounded strictly convex smooth domains in $\mathbb{R}^{2}$.

The above upper bound of $\nabla u$ was localized and extended to higher dimensions by Bao, Li and Yin in [4], where they considered problem (1.2) and proved

$$
\|\nabla u\|_{L^{\infty}(\widetilde{\Omega})} \leq C \varepsilon^{-1 / 2}\|\varphi\|_{C^{2}(\partial \Omega)}, \quad \text { when } n \geq 2 .
$$

The upper bound is optimal for $n=2$ as mentioned earlier. For dimensions $n \geq 3$, the upper bound was recently improved by Li and Yang [12] to

$$
\|\nabla u\|_{L^{\infty}(\widetilde{\Omega})} \leq C \varepsilon^{-1 / 2+\beta}\|\varphi\|_{C^{2}(\partial \Omega)}, \quad \text { when } n \geq 3,
$$

for some $\beta>0$.

Yun [16] considered the problem (1.3) in $\mathbb{R}^{3}$, with unit disks

$$
D_{1}=B_{1}(0,0,1+\varepsilon / 2), \quad D_{2}=B_{1}(0,0,-1-\varepsilon / 2),
$$

and a harmonic function $H$. He proved that for some positive constant $C$ independent of $\varepsilon$,

$$
\max _{\left|x_{3}\right| \leq \varepsilon / 2}\left|\nabla u\left(0,0, x_{3}\right)\right| \leq C \varepsilon^{\frac{\sqrt{2}-2}{2}} .
$$

He also showed that this upper bound of $|\nabla u|$ on the $\varepsilon$-segment connecting $D_{1}$ and $D_{2}$ is optimal for $H(x) \equiv x_{1}$.

In this paper, we assume that for some $m \in[2, \infty)$ and a small universal constant $R_{0}$, the portions of $\partial D_{1}^{*}$ and $\partial D_{2}^{*}$ in $\left[-R_{0}, R_{0}\right]^{n}$ are respectively the graphs of two $C^{2}$ functions $f$ and $g$ in terms of $x^{\prime}$, and

$$
\begin{array}{ll}
f\left(0^{\prime}\right)=g\left(0^{\prime}\right)=0, \quad \nabla f\left(0^{\prime}\right)=\nabla g\left(0^{\prime}\right)=0, \\
\lambda_{1}\left|x^{\prime}\right|^{m} \leq(f-g)\left(x^{\prime}\right) \leq \lambda_{2}\left|x^{\prime}\right|^{m} & \text { for } 0<\left|x^{\prime}\right|<R_{0}, \\
\left|\nabla(f-g)\left(x^{\prime}\right)\right| \leq \lambda_{3}\left|x^{\prime}\right|^{m-1} & \text { for } 0<\left|x^{\prime}\right|<R_{0},
\end{array}
$$


for some $\lambda_{1}, \lambda_{2}, \lambda_{3}>0$. Let $a(x) \in C^{\alpha}(\overline{\widetilde{\Omega}})$, for some $\alpha \in(0,1)$, be a symmetric, positive definite matrix function satisfying

$$
\lambda \leq a(x) \leq \Lambda \text { for } x \in \widetilde{\Omega},
$$

for some positive constants $\lambda, \Lambda$. Let $v=\left(v_{1}, \cdots, v_{n}\right)$ denote the unit normal vector on $\partial D_{1}$ and $\partial D_{2}$, pointing towards the interior of $D_{1}$ and $D_{2}$. We consider the following insulated conductivity problem:

$$
\begin{cases}-\partial_{i}\left(a^{i j} \partial_{j} u\right)=0 & \text { in } \widetilde{\Omega} \\ a^{i j} \partial_{j} u v_{i}=0 & \text { on } \partial\left(D_{1} \cup D_{2}\right) \\ u=\varphi & \text { on } \partial \Omega,\end{cases}
$$

where $\varphi \in C^{2}(\partial \Omega)$ is given. For $0<r \leq R_{0}$, we denote

$$
\begin{aligned}
& \Omega_{x_{0}, r}:=\left\{\left(x^{\prime}, x_{n}\right) \in \widetilde{\Omega}\left|-\frac{\varepsilon}{2}+g\left(x^{\prime}\right)<x_{n}<\frac{\varepsilon}{2}+f\left(x^{\prime}\right),\right| x^{\prime}-x_{0}^{\prime} \mid<r\right\}, \\
& \Gamma_{+}:=\left\{x_{n}=\frac{\varepsilon}{2}+f\left(x^{\prime}\right),\left|x^{\prime}\right|<R_{0}\right\}, \quad \Gamma_{-}:=\left\{x_{n}=-\frac{\varepsilon}{2}+g\left(x^{\prime}\right),\left|x^{\prime}\right|<R_{0}\right\} .
\end{aligned}
$$

Since the blow-up of gradient can only occur in the narrow region between $D_{1}$ and $D_{2}$, we will focus on the following problem near the origin:

$$
\begin{cases}-\partial_{i}\left(a^{i j} \partial_{j} u\right)=0 & \text { in } \Omega_{0, R_{0}} \\ a^{i j} \partial_{j} u v_{i}=0 & \text { on } \Gamma_{+} \cup \Gamma_{-},\end{cases}
$$

where $v=\left(v_{1}, \cdots, v_{n}\right)$ denotes the unit normal vector on $\Gamma_{+}$and $\Gamma_{-}$, pointing upward and downward respectively.

Theorem 1.1. Let $m, \Gamma_{+}, \Gamma_{-}, a, \alpha$ be as above, and let $u \in H^{1}\left(\Omega_{0, R_{0}}\right)$ be a solution of (1.9). There exist positive constants $r_{0}, \beta$ and $C$ depending only on $n, m, \lambda, \Lambda, R_{0}, \alpha, \lambda_{1}, \lambda_{2}, \lambda_{3}$, $\|f\|_{C^{2}\left(\left\{\left|x^{\prime}\right| \leq R_{0}\right\}\right)},\|g\|_{C^{2}\left(\left\{\left|x^{\prime}\right| \leq R_{0}\right\}\right)}$ and $\|a\|_{C^{\alpha}\left(\Omega_{0, R_{0}}\right)}$, such that

$$
\left|\nabla u\left(x_{0}\right)\right| \leq \begin{cases}C\|u\|_{L^{\infty}\left(\Omega_{0, R_{0}}\right)}\left(\varepsilon+\left|x_{0}^{\prime}\right|^{m}\right)^{-1 / m}, & \text { when } n=2, \\ C\|u\|_{L^{\infty}\left(\Omega_{0, R_{0}}\right)}\left(\varepsilon+\left|x_{0}^{\prime}\right|^{m}\right)^{-1 / m+\beta}, & \text { when } n \geq 3,\end{cases}
$$

for all $x_{0} \in \Omega_{0, r_{0}}$ and $\varepsilon \in(0,1)$.

Remark 1.1. For $m=2$, (1.10) was proved in [4] and [12] for $n=2$ and $n \geq 3$, respectively.

Let $u \in H^{1}(\widetilde{\Omega})$ be a weak solution of (1.7). By the maximum principle and the gradient estimates of solutions of elliptic equations,

$$
\begin{aligned}
& \|u\|_{L^{\infty}(\widetilde{\Omega})} \leq\|\varphi\|_{L^{\infty}(\partial \Omega)}, \\
& \|\nabla u\|_{L^{\infty}\left(\widetilde{\Omega} \backslash \Omega_{0, r_{0}}\right)} \leq C\|\varphi\|_{C^{2}(\partial \Omega)} .
\end{aligned}
$$

Therefore, a corollary of Theorem 1.1 is as follows. 
Corollary 1.1. Let $u \in H^{1}(\widetilde{\Omega})$ be a weak solution of (1.7). There exist positive constants $\beta$ and $C$ depending only on $n, m, \lambda, \Lambda, R_{0}, \alpha, \lambda_{1}, \lambda_{2}, \lambda_{3},\left\|\partial D_{1}\right\|_{C^{2}},\left\|\partial D_{2}\right\|_{C^{2}},\|\partial \Omega\|_{C^{2}}$, and $\|a\|_{C^{\alpha}(\widetilde{\Omega})^{\prime}}$ such that

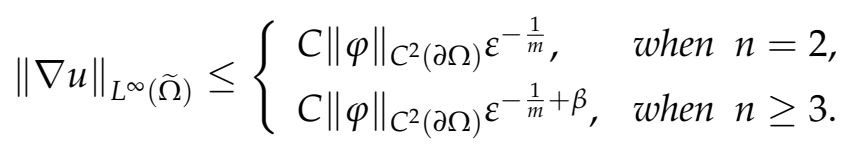

\section{Proof of Theorem 1.1}

Our proof of Theorem 1.1 is an adaption of the arguments in our earlier paper [12] for $m=2$, and follows closely the arguments there.

We fix a $\gamma \in(0,1)$, and let $r_{0}>0$ denote a constant depending only on $n, m, \gamma, R_{0}, \lambda_{1}$, $\lambda_{2},\|f\|_{C^{2}}$ and $\|g\|_{C^{2}}$, whose value will be fixed in the proof. For any $x_{0} \in \Omega_{0, r_{0}}$, we define

$$
\delta:=\left(\varepsilon+\left|x_{0}^{\prime}\right|^{m}\right)^{\frac{1}{m}} .
$$

We will always consider $0<\varepsilon \leq r_{0}^{m}$. First, we require $r_{0}$ small so that for $\left|x_{0}^{\prime}\right|<r_{0}$,

$$
10 \delta<\delta^{1-\gamma}<\frac{R_{0}}{4}
$$

Lemma 2.1. For $n \geq 3$, there exists a small $r_{0}$, depending only on $n, m, \gamma$, and $R_{0}$, such that for any $x_{0} \in \Omega_{0, r_{0}}, 5\left|x_{0}^{\prime}\right|<r<\delta^{1-\gamma}$, if $u \in H^{1}\left(\Omega_{x_{0}, 2 r} \backslash \Omega_{x_{0}, r / 4}\right)$ is a positive solution to the equation

$$
\left\{\begin{array}{lll}
-\partial_{i}\left(a^{i j}(x) \partial_{j} u(x)\right)=0 & \text { in } \Omega_{x_{0}, 2 r} \backslash \Omega_{x_{0}, r / 4} \\
a^{i j}(x) \partial_{j} u(x) v_{i}(x)=0 & \text { on }\left(\Gamma_{+} \cup \Gamma_{-}\right) \cap \overline{\Omega_{x_{0}, 2 r} \backslash \Omega_{x_{0}, r / 4}}
\end{array}\right.
$$

then

$$
\sup _{\Omega_{x_{0}, r} \backslash \Omega_{x_{0}, r / 2}} u \leq C \inf _{\Omega_{x_{0}, r} \backslash \Omega_{x_{0}, r / 2}} u,
$$

for some constant $C>0$ depending only on $n, m, \lambda, \Lambda, R_{0}, \lambda_{1}, \lambda_{2},\|f\|_{C^{2}}$ and $\|g\|_{C^{2}}$ but independent of $r$ and $u$.

Proof. We only need to prove (2.2) for $\left|x_{0}^{\prime}\right|>0$, since the $\left|x_{0}^{\prime}\right|=0$ case follows from the result for $\left|x_{0}^{\prime}\right|>0$ and then sending $\left|x_{0}^{\prime}\right|$ to 0 . We denote

$$
h_{r}:=\varepsilon+f\left(x_{0}^{\prime}-\frac{r}{4} \frac{x_{0}^{\prime}}{\left|x_{0}^{\prime}\right|}\right)-g\left(x_{0}^{\prime}-\frac{r}{4} \frac{x_{0}^{\prime}}{\left|x_{0}^{\prime}\right|}\right),
$$

and perform a change of variables by setting

$$
\left\{\begin{array}{l}
y^{\prime}=x^{\prime}-x_{0}^{\prime} \\
y_{n}=2 h_{r}\left(\frac{x_{n}-g\left(x^{\prime}\right)+\varepsilon / 2}{\varepsilon+f\left(x^{\prime}\right)-g\left(x^{\prime}\right)}-\frac{1}{2}\right), \quad\left(x^{\prime}, x_{n}\right) \in \Omega_{x_{0}, 2 r} \backslash \Omega_{x_{0}, r / 4} .
\end{array}\right.
$$


This change of variables maps the domain $\Omega_{x_{0}, 2 r} \backslash \Omega_{x_{0}, r / 4}$ to an annular cylinder of height $h_{r}$, denoted by $Q_{2 r, h_{r}} \backslash Q_{r / 4, h_{r}}$, where

$$
Q_{s, t}:=\left\{y=\left(y^{\prime}, y_{n}\right) \in \mathbb{R}^{n}|| y^{\prime}|<s,| y_{n} \mid<t\right\},
$$

for $s, t>0$. We will show that the Jacobian matrix of the change of variables (2.3), denoted by $\partial_{x} y$, and its inverse matrix $\partial_{y} x$ satisfy

$$
\left|\left(\partial_{x} y\right)^{i j}\right| \leq C, \quad\left|\left(\partial_{y} x\right)^{i j}\right| \leq C \quad \text { for } y \in Q_{2 r, h_{r}} \backslash Q_{r / 4, h_{r}}
$$

where $C>0$ depends only on $n, m, R_{0}, \lambda_{1}, \lambda_{2},\|f\|_{C^{2}}$ and $\|g\|_{C^{2}}$.

Let $v(y)=u(x)$, then $v$ satisfies

$$
\begin{cases}-\partial_{i}\left(b^{i j}(y) \partial_{j} v(y)\right)=0 & \text { in } Q_{2 r, h_{r}} \backslash Q_{r / 4, h_{r}} \\ b^{n j}(y) \partial_{j} v(y)=0 & \text { on }\left\{y_{n}=-h_{r}\right\} \cup\left\{y_{n}=h_{r}\right\},\end{cases}
$$

where the matrix $\left(b^{i j}(y)\right)$ is given by

$$
\left(b^{i j}(y)\right)=\frac{\left(\partial_{x} y\right)\left(a^{i j}\right)\left(\partial_{x} y\right)^{t}}{\operatorname{det}\left(\partial_{x} y\right)},
$$

$\left(\partial_{x} y\right)^{t}$ is the transpose of $\partial_{x} y$.

It is easy to see that (2.5) implies, using $\lambda \leq\left(a^{i j}\right) \leq \Lambda$,

$$
\frac{\lambda}{C} \leq\left(b^{i j}(y)\right) \leq C \Lambda \text { for } y \in Q_{2 r, h_{r}} \backslash Q_{r / 4, h_{r}},
$$

for some constant $C>0$ depending only on $n, m, R_{0}, \lambda_{1}, \lambda_{2},\|f\|_{C^{2}}$ and $\|g\|_{C^{2}}$.

In the following and throughout this section, we will denote $A \sim B$, if there exists a positive universal constant $C$, which might depend on $n, m, \lambda, \Lambda, R_{0}, \lambda_{1}, \lambda_{2},\|f\|_{C^{2}}$ and $\|g\|_{C^{2}}$, but not depend on $\varepsilon$, such that $C^{-1} B \leq A \leq C B$.

From (2.3), one can compute that

$$
\begin{aligned}
& \left(\partial_{x} y\right)^{i i}=1 \quad \text { for } 1 \leq i \leq n-1, \\
& \left(\partial_{x} y\right)^{n n}=\frac{2 h_{r}}{\varepsilon+f\left(x_{0}^{\prime}+y^{\prime}\right)-g\left(x_{0}^{\prime}+y^{\prime}\right)}, \\
& \left(\partial_{x} y\right)^{n i}=-\frac{2 h_{r} \partial_{i} g\left(x_{0}^{\prime}+y^{\prime}\right)+2 y_{n}\left[\partial_{i} f\left(x_{0}^{\prime}+y^{\prime}\right)-\partial_{i} g\left(x_{0}^{\prime}+y^{\prime}\right)\right]}{\varepsilon+f\left(x_{0}^{\prime}+y^{\prime}\right)-g\left(x_{0}^{\prime}+y^{\prime}\right)} \quad \text { for } 1 \leq i \leq n-1, \\
& \left(\partial_{x} y\right)^{i j}=0 \quad \text { for } 1 \leq i \leq n-1, \quad j \neq i .
\end{aligned}
$$

By (1.6b), one can see that

$$
h_{r} \sim \varepsilon+\left|x_{0}^{\prime}-\frac{r}{4} \frac{x_{0}^{\prime}}{\left|x_{0}^{\prime}\right|}\right|^{m} .
$$


Since $\left|y_{n}\right| \leq h_{r}$, by using (1.6a) and (1.6b), we have that, for $1 \leq i \leq n-1$,

$$
\begin{aligned}
\left|\left(\partial_{x} y\right)^{n i}\right| & \leq C \frac{h_{r}\left|\partial_{i} g\left(x_{0}^{\prime}+y^{\prime}\right)\right|+h_{r}\left[\left|\partial_{i} f\left(x_{0}^{\prime}+y^{\prime}\right)\right|+\left|\partial_{i} g\left(x_{0}^{\prime}+y^{\prime}\right)\right|\right]}{\varepsilon+f\left(x_{0}^{\prime}+y^{\prime}\right)-g\left(x_{0}^{\prime}+y^{\prime}\right)} \\
& \leq C \frac{h_{r}}{\varepsilon+f\left(x_{0}^{\prime}+y^{\prime}\right)-g\left(x_{0}^{\prime}+y^{\prime}\right)}\left[\left|\partial_{i} f\left(x_{0}^{\prime}+y^{\prime}\right)\right|+\left|\partial_{i} g\left(x_{0}^{\prime}+y^{\prime}\right)\right|\right] \\
& \leq C \frac{\varepsilon+\left|x_{0}^{\prime}-\frac{r}{4} \frac{x_{0}^{\prime}}{\left|x_{0}^{\prime}\right|}\right|^{m}}{\varepsilon+\left|x_{0}^{\prime}+y^{\prime}\right|^{m}}\left|x_{0}^{\prime}+y^{\prime}\right| .
\end{aligned}
$$

Since $r / 4<\left|y^{\prime}\right|<2 r<2 \delta^{1-\gamma}$ and $\left|x_{0}^{\prime}\right|<\delta$, we can estimate

$$
\left|\left(\partial_{x} y\right)^{n i}\right| \leq C\left|x_{0}^{\prime}+y^{\prime}\right| \leq C\left(\left|x_{0}^{\prime}\right|+\left|y^{\prime}\right|\right) \leq C \delta^{1-\gamma} .
$$

Next, we will show that

$$
\left(\partial_{x} y\right)^{n n} \sim 1 \text { for } y \in Q_{2 r, h_{r}} \backslash Q_{r / 4, h_{r}} .
$$

Indeed, by (1.6b), we have

$$
\left(\partial_{x} y\right)^{n n}=\frac{2 h_{r}}{\varepsilon+f\left(x_{0}^{\prime}+y^{\prime}\right)-g\left(x_{0}^{\prime}+y^{\prime}\right)} \sim \frac{\varepsilon+\left|x_{0}^{\prime}-\frac{r}{4} \frac{x_{0}^{\prime}}{\left|x_{0}^{\prime}\right|}\right|^{m}}{\varepsilon+\left|x_{0}^{\prime}+y^{\prime}\right|^{m}} .
$$

Since $\left|y^{\prime}\right|>r / 4$, it is easy to see

$$
\left(\partial_{x} y\right)^{n n} \leq C \frac{\varepsilon+\left|x_{0}^{\prime}-\frac{r}{4} \frac{x_{0}^{\prime}}{\left|x_{0}^{\prime}\right|}\right|^{m}}{\varepsilon+\left|x_{0}^{\prime}+y^{\prime}\right|^{m}} \leq C .
$$

On the other hand, since $\left|y^{\prime}\right|<2 r$ and $\left|x_{0}^{\prime}\right|<r / 5$, we have

$$
\begin{aligned}
& \varepsilon+\left|x_{0}^{\prime}-\frac{r}{4} \frac{x_{0}^{\prime}}{\left|x_{0}^{\prime}\right|}\right|^{m} \geq \varepsilon+\left(\left|\frac{r}{4} \frac{x_{0}^{\prime}}{\left|x_{0}^{\prime}\right|}\right|-\left|x_{0}^{\prime}\right|\right)^{m} \geq \varepsilon+\left(\frac{r}{4}-\frac{r}{5}\right)^{m} \geq \frac{1}{C}\left(\varepsilon+r^{m}\right), \\
& \varepsilon+\left|x_{0}^{\prime}+y^{\prime}\right|^{m} \leq \varepsilon+m\left|x_{0}^{\prime}\right|^{m}+m\left|y^{\prime}\right|^{m} \leq C\left(\varepsilon+r^{m}\right) .
\end{aligned}
$$

Therefore,

$$
\left(\partial_{x} y\right)^{n n} \geq \frac{1}{C} \frac{\varepsilon+\left|x_{0}^{\prime}-\frac{r}{4} \frac{x_{0}^{\prime}}{\left|x_{0}^{\prime}\right|}\right|^{m}}{\varepsilon+\left|x_{0}^{\prime}+y^{\prime}\right|^{m}} \geq \frac{1}{C}
$$

and (2.9) is verified.

We have shown $\left(\partial_{x} y\right)^{i i} \sim 1$ for all $i=1, \cdots, n$, and $\left|\left(\partial_{x} y\right)^{i j}\right| \leq C \delta^{(1-\gamma)}$ for $i \neq j$. We further require $r_{0}$ to be small enough so that off-diagonal entries of $\partial_{x} y$ are small. Therefore (2.5) follows. As mentioned earlier, (2.8) follows from (2.5). 
Now we define, for any integer $l$,

$$
A_{l}:=\left\{y \in \mathbb{R}^{n}\left|\frac{r}{4}<\right| y^{\prime} \mid<2 r,(l-1) h_{r}<z_{n}<(l+1) h_{r}\right\} .
$$

Note that $A_{0}=Q_{2 r, h_{r}} \backslash Q_{r / 4, h_{r}}$. For any $l \in \mathbb{Z}$, we define a new function $\tilde{v}$ by

$$
\tilde{v}(y):=v\left(y^{\prime},(-1)^{l}\left(y_{n}-2 l h_{r}\right)\right), \quad \forall y \in A_{l} .
$$

We also define the corresponding coefficients, for $k=1,2, \cdots, n-1$,

$$
\tilde{b}^{n k}(y)=\tilde{b}^{k n}(y):=(-1)^{l} b^{n k}\left(y^{\prime},(-1)^{l}\left(y_{n}-2 l h_{r}\right)\right), \quad \forall y \in A_{l},
$$

and for other indices,

$$
\tilde{b}^{i j}(y):=b^{i j}\left(y^{\prime},(-1)^{l}\left(y_{n}-2 l h_{r}\right)\right), \quad \forall y \in A_{l} .
$$

Therefore, $\tilde{v}(y)$ and $\tilde{b}^{i j}(y)$ are defined in the infinite cylinder shell $Q_{2 r, \infty} \backslash Q_{r / 4, \infty}$. By (2.6), $\tilde{v} \in H^{1}\left(Q_{2 r, \infty} \backslash Q_{r / 4, \infty}\right)$ satisfies

$$
-\partial_{i}\left(\tilde{b}^{i j}(y) \partial_{j} \tilde{\mathcal{v}}(y)\right)=0 \text { in } Q_{2 r, \infty} \backslash Q_{r / 4, \infty} .
$$

Note that for any $l \in \mathbb{Z}$ and $y \in A_{l}, \tilde{b}(y)=\left(\tilde{b}^{i j}(y)\right)$ is orthogonally conjugated to $b\left(y^{\prime},(-1)^{l}\left(y_{n}-2 l h_{r}\right)\right)$. Hence, by (2.8), we have

$$
\frac{\lambda}{C} \leq \tilde{b}(y) \leq C \Lambda \quad \text { for } y \in Q_{2 r, \infty} \backslash Q_{r / 4, \infty} .
$$

We restrict the domain to be $Q_{2 r, r} \backslash Q_{r / 4, r}$, and make the change of variables $z=y / r$. Set $\bar{v}(z)=\tilde{v}(y), \bar{b}^{i j}(z)=\tilde{b}^{i j}(y)$, we have

$$
\begin{array}{ll}
-\partial_{i}\left(\bar{b}^{i j}(z) \partial_{j} \bar{v}(z)\right)=0 & \text { in } \quad Q_{2,1} \backslash Q_{1 / 4,1}, \\
\frac{\lambda}{C} \leq \bar{b}(z) \leq C \Lambda & \text { for } z \in Q_{2,1} \backslash Q_{1 / 4,1} .
\end{array}
$$

Then by the Harnack inequality for uniformly elliptic equations of divergence form, see e.g., [8, Theorem 8.20], there exists a constant $C$ depending only on $n, m, \lambda, \Lambda, R_{0}, \lambda_{1}, \lambda_{2}$, $\|f\|_{C^{2}}$ and $\|g\|_{C^{2}}$, such that

$$
\sup _{Q_{1,1 / 2} \backslash Q_{1 / 2,1 / 2}} \bar{v} \leq C \inf _{Q_{1,1 / 2} \backslash Q_{1 / 2,1 / 2}} \bar{v} .
$$

In particular, we have

$$
\sup _{Q_{1, h_{r} / r} \backslash Q_{1 / 2, h_{r} / r}} \bar{v} \leq C \underset{Q_{1, h_{r} / r} \backslash Q_{1 / 2, h_{r} / r}}{\inf _{1}} \bar{v},
$$

which is (2.2) after reversing the change of variables. 
Remark 2.1. Lemma 2.1 does not hold for dimension $n=2$, since $Q_{2,1} \backslash Q_{1 / 4,1} \subset \mathbb{R}^{2}$ is the union of two disjoint rectangular domains, and the Harnack inequality cannot be applied on it. Therefore, we will separate the cases $n=2$ and $n \geq 3$ in our proof of Theorem 1.1.

For any domain $A \subset \widetilde{\Omega}$, we denote the oscillation of $u$ in $\mathrm{A}$ by $\operatorname{osc}_{A} u:=\sup _{A} u-$ $\inf _{A} u$. Using Lemma 2.1, we obtain a decay of $\operatorname{osc}_{\Omega_{x_{0}, \delta}} u$ in $\delta$ as follows.

Lemma 2.2. For $n \geq 3$, let $u$ be a solution of (1.9). For any $x_{0} \in \Omega_{0, r_{0}}$, where $r_{0}$ is as in Lemma 2.1 , there exist positive constants $\sigma$ and $C$, depending only on $n, m, \lambda, \Lambda, R_{0}, \lambda_{1}, \lambda_{2},\|f\|_{C^{2}}$ and $\|g\|_{C^{2}}$ such that

$$
\operatorname{osc}_{\Omega_{x_{0}, \delta}} u \leq C\|u\|_{L^{\infty}\left(\Omega_{x_{0}, \delta^{1-\gamma}}\right)} \delta^{\gamma \sigma} .
$$

Proof. For simplicity, we drop the $x_{0}$ subscript and denote $\Omega_{r}=\Omega_{x_{0}, r}$ in this proof. Let $5\left|x_{0}^{\prime}\right|<r<\delta^{1-\gamma}$ and $u_{1}=\sup _{\Omega_{2 r}} u-u, u_{2}=u-\inf _{\Omega_{2 r}} u$. By Lemma 2.1, we have

$$
\sup _{\Omega_{r} \backslash \Omega_{r / 2}} u_{1} \leq C_{1} \inf _{\Omega_{r} \backslash \Omega_{r / 2}} u_{1}, \quad \sup _{\Omega_{r} \backslash \Omega_{r / 2}} u_{2} \leq C_{1} \inf _{\Omega_{r} \backslash \Omega_{r / 2}} u_{2}
$$

where $C_{1}>1$ is a constant independent of $r$. Since both $u_{1}$ and $u_{2}$ satisfy Eq. (1.9), by the maximum principle,

$$
\sup _{\Omega_{r} \backslash \Omega_{r / 2}} u_{i}=\sup _{\Omega_{r}} u_{i}, \quad \inf _{\Omega_{r} \backslash \Omega_{r / 2}} u_{i}=\inf _{\Omega_{r}} u_{i}
$$

for $i=1,2$. Therefore,

$$
\sup _{\Omega_{r}} u_{1} \leq C_{1} \inf _{\Omega_{r}} u_{1}, \quad \sup _{\Omega_{r}} u_{2} \leq C_{1} \inf _{\Omega_{r}} u_{2}
$$

Adding up the above two inequalities, we have

$$
\operatorname{osc}_{\Omega_{r}} u \leq\left(\frac{C_{1}-1}{C_{1}+1}\right) \operatorname{osc}_{\Omega_{2 r}} u \text {. }
$$

Now we take $\sigma>0$ such that $2^{-\sigma}=\frac{C_{1}-1}{C_{1}+1}$, then

$$
\operatorname{osc}_{\Omega_{r}} u \leq 2^{-\sigma} \operatorname{osc}_{\Omega_{2 r}} u \text {. }
$$

We start with $r=r_{0}=\delta^{1-\gamma} / 2$, and set $r_{i+1}=r_{i} / 2$. Keep iterating (2.11) $k+1$ times, where $k$ satisfies $5 \delta \leq r_{k}<10 \delta$, we will have

$$
\operatorname{osc}_{\Omega_{\delta}} u \leq \operatorname{osc}_{\Omega_{r_{k}}} u \leq 2^{-(k+1) \sigma} \operatorname{osc}_{\Omega_{2 r_{0}}} u \leq 2^{1-(k+1) \sigma}\|u\|_{L^{\infty}\left(\Omega_{\delta^{1-\gamma}}\right)} .
$$

Since

$$
10 \delta>r^{k}=2^{-k} r_{0}=2^{-(k+1)} \delta^{1-\gamma},
$$

we have

$$
2^{-(k+1)}<10 \delta^{\gamma}
$$

and hence (2.10) follows immediately. 
Proof of Theorem 1.1. First we consider the case when $n \geq 3$. Let $u \in H^{1}\left(\Omega_{0, R_{0}}\right)$ be a solution of (1.9). For $x_{0} \in \Omega_{0, r_{0}}$, we have, using Lemma 2.2,

$$
\left\|u-u_{0}\right\|_{L^{\infty}\left(\Omega_{x_{0}, \delta}\right)} \leq C\|u\|_{L^{\infty}\left(\Omega_{x_{0}, \delta^{1-\gamma}} \delta^{\gamma \sigma}\right.}
$$

for some constant $u_{0}$. We denote $v:=u-u_{0}$, and $v$ satisfies the same equation (1.9). We work on the domain $\Omega_{x_{0}, \delta / 4}$, and perform a change of variables by setting

$$
\left\{\begin{array}{l}
y^{\prime}=\delta^{-1}\left(x^{\prime}-x_{0}^{\prime}\right), \\
y_{n}=\delta^{-1} x_{n}
\end{array}\right.
$$

The domain $\Omega_{x_{0}, \delta / 4}$ becomes

$$
\left\{y \in \mathbb{R}^{n}|| y^{\prime} \mid \leq \frac{1}{4}, \delta^{-1}\left(-\frac{1}{2} \varepsilon+g\left(x_{0}^{\prime}+\delta y^{\prime}\right)\right)<y_{n}<\delta^{-1}\left(\frac{1}{2} \varepsilon+f\left(x_{0}^{\prime}+\delta y^{\prime}\right)\right)\right\} .
$$

We make a change of variables again by

$$
\left\{\begin{array}{l}
z^{\prime}=4 y^{\prime} \\
z_{n}=2 \delta^{m-1}\left(\frac{\delta y_{n}-g\left(x_{0}^{\prime}+\delta y^{\prime}\right)+\varepsilon / 2}{\varepsilon+f\left(x_{0}^{\prime}+\delta y^{\prime}\right)-g\left(x_{0}^{\prime}+\delta y^{\prime}\right)}-\frac{1}{2}\right) .
\end{array}\right.
$$

Now the domain in $z$-variables becomes a thin plate $Q_{1, \delta^{m-1}}$, where $Q_{s, t}$ is defined as in (2.4). Let $w(z)=v(x)$, then $w$ satisfies

$$
\begin{cases}-\partial_{i}\left(b^{i j}(z) \partial_{j} w(z)\right)=0 & \text { in } Q_{1, \delta^{m-1}} \\ b^{n j}(z) \partial_{j} w(z)=0 & \text { on }\left\{z_{n}=-\delta\right\} \cup\left\{z_{n}=\delta\right\},\end{cases}
$$

where the matrix $b(z)=\left(b^{i j}(z)\right)$ is given by

$$
\left(b^{i j}(z)\right)=\frac{\left(\partial_{y} z\right)\left(a^{i j}\right)\left(\partial_{y} z\right)^{t}}{\operatorname{det}\left(\partial_{y} z\right)} .
$$

Similar to the proof of Lemma 2.1, we will show that the Jacobian matrix of the change of variables (2.14), denoted by $\partial_{y} z$, and its inverse matrix $\partial_{z} y$ satisfy

$$
\left|\left(\partial_{y} z\right)^{i j}\right| \leq C, \quad\left|\left(\partial_{z} y\right)^{i j}\right| \leq C \quad \text { for } z \in Q_{1, \delta^{m-1}},
$$

where $C>0$ depends only on $n, \kappa, R_{0}, \lambda_{1}, \lambda_{2},\|f\|_{C^{2}}$ and $\|g\|_{C^{2}}$. This leads to

$$
\frac{\lambda}{C} \leq b(z) \leq C \Lambda \quad \text { for } z \in Q_{1, \delta^{m-1}}
$$


From (2.14), one can compute that

$$
\begin{aligned}
& \left(\partial_{y} z\right)^{i i}=4 \quad \text { for } 1 \leq i \leq n-1, \\
& \left(\partial_{y} z\right)^{n n}=\frac{2 \delta^{m}}{\varepsilon+f\left(x_{0}^{\prime}+\delta z^{\prime} / 4\right)-g\left(x_{0}^{\prime}+\delta z^{\prime} / 4\right)}, \\
& \left(\partial_{y} z\right)^{n i}=-\frac{2 \delta^{m} \partial_{i} g\left(x_{0}^{\prime}+\delta z^{\prime} / 4\right)+\left(z_{n}+\delta^{m-1}\right) \delta\left[\partial_{i} f\left(x_{0}^{\prime}+\delta z^{\prime} / 4\right)-\partial_{i} g\left(x_{0}^{\prime}+\delta z^{\prime} / 4\right)\right]}{\varepsilon+f\left(x_{0}^{\prime}+\delta z^{\prime} / 4\right)-g\left(x_{0}^{\prime}+\delta z^{\prime} / 4\right)} \\
& \quad \text { for } 1 \leq i \leq n-1, \\
& \left(\partial_{y} z\right)^{i j}=0 \quad \text { for } 1 \leq i \leq n-1, \quad j \neq i .
\end{aligned}
$$

First we will show that

$$
\left(\partial_{y} z\right)^{n n} \sim 1 \text { for } z \in Q_{1, \delta^{m-1}} .
$$

Since $\left|z^{\prime}\right|<1$ and $\left|x_{0}^{\prime}\right|<\delta$, it is easy to see that

$$
\left(\partial_{y} z\right)^{n n} \geq \frac{1}{C} \frac{\delta^{m}}{\varepsilon+\left|x_{0}^{\prime}+\delta z^{\prime} / 4\right|^{m}} \geq \frac{1}{C} \frac{\delta^{m}}{\varepsilon+C \delta^{m}} \geq \frac{1}{C} \quad \text { for } \quad z \in Q_{1, \delta^{m-1}} .
$$

On the other hand, when $\left|x_{0}^{\prime}\right| \leq \varepsilon^{\frac{1}{m}}$, we have $\delta \leq(2 \varepsilon)^{\frac{1}{m}}$, and hence

$$
\left(\partial_{y} z\right)^{n n} \leq \frac{C \delta^{m}}{\varepsilon+\left|x_{0}^{\prime}+\delta z^{\prime} / 4\right|^{m}} \leq \frac{C \varepsilon}{\varepsilon+\left|x_{0}^{\prime}+\delta z^{\prime} / 4\right|^{m}} \leq C \quad \text { for } \quad z \in Q_{1, \delta^{m-1}} .
$$

When $\left|x_{0}^{\prime}\right| \geq \varepsilon^{\frac{1}{m}}$, we have $\left|\delta z^{\prime} / 4\right| \leq\left|x_{0}^{\prime}\right| / 2$, and hence

$$
\begin{aligned}
\left(\partial_{y} z\right)^{n n} & \leq \frac{C \delta^{m}}{\varepsilon+\left|x_{0}^{\prime}+\delta z^{\prime} / 4\right|^{m}} \leq \frac{C \delta^{m}}{\varepsilon+\left(\left|x_{0}^{\prime}\right|-\left|\delta z^{\prime} / 4\right|\right)^{m}} \\
& \leq \frac{2 \delta^{m}}{\varepsilon+\left(\left|x_{0}^{\prime}\right| / 2\right)^{m}} \leq C \text { for } z \in Q_{1, \delta^{m-1}} .
\end{aligned}
$$

Therefore, (2.19) is verified. Since $\left|z_{n}\right|<\delta^{m-1},\left|z^{\prime}\right|<1$ and $\left|x_{0}^{\prime}\right|<\delta$, by (1.6a) and (1.6b), for $1 \leq i \leq n-1$,

$$
\begin{aligned}
\left|\left(\partial_{y} z\right)^{n i}\right| & \leq \frac{2 \delta^{m}\left|\partial_{i} g\left(x_{0}^{\prime}+\delta z^{\prime} / 4\right)\right|+2 \delta^{m}\left[\left|\partial_{i} f\left(x_{0}^{\prime}+\delta z^{\prime} / 4\right)\right|+\left|\partial_{i} g\left(x_{0}^{\prime}+\delta z^{\prime} / 4\right)\right|\right]}{\varepsilon+f\left(x_{0}^{\prime}+\delta z^{\prime} / 4\right)-g\left(x_{0}^{\prime}+\delta z^{\prime} / 4\right)} \\
& \leq \frac{C \delta^{m}}{\varepsilon+f\left(x_{0}^{\prime}+\delta z^{\prime} / 4\right)-g\left(x_{0}^{\prime}+\delta z^{\prime} / 4\right)}\left[\left|\partial_{i} f\left(x_{0}^{\prime}+\delta z^{\prime} / 4\right)\right|+\left|\partial_{i} g\left(x_{0}^{\prime}+\delta z^{\prime} / 4\right)\right|\right] \\
& \leq C \frac{\delta^{m}}{\varepsilon+\left|x_{0}^{\prime}+\delta z^{\prime} / 4\right|^{m}}\left|x_{0}^{\prime}+\delta z^{\prime} / 4\right| \\
& \leq C\left(\left|x_{0}^{\prime}\right|+\delta\left|z^{\prime}\right|\right) \leq C \delta
\end{aligned}
$$

where in the last line, we have used the same arguments in showing $\left(\partial_{y} z\right)^{n n} \leq C$ earlier. 
We have shown $\left(\partial_{y} z\right)^{i i} \sim 1$ for all $i=1, \cdots, n$, and $\left|\left(\partial_{y} z\right)^{i j}\right| \leq C \delta$ for $i \neq j$. We further require $r_{0}$ to be small enough so that off-diagonal entries are small. Therefore (2.17) follows. As mentioned earlier, (2.18) follows from (2.17).

Next, we will show

$$
\|b\|_{C^{\alpha}\left(\bar{Q}_{1, \delta m-1}\right)} \leq C
$$

for some $C>0$ depending only on $n, m, R_{0}, \lambda_{1}, \lambda_{2}, \lambda_{3},\|f\|_{C^{2}},\|g\|_{C^{2}}$ and $\|a\|_{C^{\alpha}}$, by showing

$$
\left|\nabla_{z}\left(\partial_{y} z\right)^{i j}(z)\right| \leq C, \quad\left|\nabla_{z} \frac{1}{\operatorname{det}\left(\partial_{y} z\right)}\right| \leq C \quad \text { for } z \in Q_{1, \delta^{m-1}} .
$$

Then (2.20) follows from (2.21), (2.16), and $\|a\|_{C^{\alpha}} \leq C$.

By a straightforward computation, we have, for any $i=1, \cdots, n-1$,

$$
\begin{aligned}
\left|\partial_{z_{i}} \frac{1}{\operatorname{det}\left(\partial_{y} z\right)}\right| & =\left|\partial_{z_{i}}\left(\frac{\varepsilon+f\left(x_{0}^{\prime}+\delta z^{\prime} / 4\right)-g\left(x_{0}^{\prime}+\delta z^{\prime} / 4\right)}{2 \cdot 4^{n-1} \delta^{m}}\right)\right| \\
& =\left|\frac{\delta\left[\partial_{i} f\left(x_{0}^{\prime}+\delta z^{\prime} / 4\right)-\partial_{i} g\left(x_{0}^{\prime}+\delta z^{\prime} / 4\right)\right]}{2 \cdot 4^{n-1} \delta^{m}}\right| \\
& \leq \frac{C}{\delta^{m-1}}\left|x_{0}^{\prime}+\delta z^{\prime} / 4\right|^{m-1} \leq C \quad \text { for } z \in Q_{1, \delta}
\end{aligned}
$$

where in the last line, (1.6b) and (1.6c) have been used. For any $i=1, \cdots, n-1$, by (1.6b) and (1.6c),

$$
\begin{aligned}
\left|\partial_{z_{i}}\left(\partial_{y} z\right)^{n n}\right| & =\left|\frac{2 \delta^{m+1}\left[\partial_{i} f\left(x_{0}^{\prime}+\delta z^{\prime} / 4\right)-\partial_{i} g\left(x_{0}^{\prime}+\delta z^{\prime} / 4\right)\right]}{\left(\varepsilon+f\left(x_{0}^{\prime}+\delta z^{\prime} / 4\right)-g\left(x_{0}^{\prime}+\delta z^{\prime} / 4\right)\right)^{2}}\right| \\
& \leq \frac{C \delta^{m+1}}{\left(\varepsilon+\left|x_{0}^{\prime}+\delta z^{\prime} / 4\right|^{m}\right)^{2}}\left|x_{0}^{\prime}+\delta z^{\prime} / 4\right|^{m-1} \\
& \leq \frac{C \delta^{m+1}\left|x_{0}^{\prime}+\delta z^{\prime} / 4\right|^{m-1}}{\delta^{2 m}} \leq C \text { for } z \in Q_{1, \delta},
\end{aligned}
$$

where in the last line, we have used the same arguments in showing $\left(\partial_{y} z\right)^{n n} \leq C$ earlier. Similar computations apply to $\partial_{z_{i}}\left(\partial_{y} z\right)^{n i}$ for $i=1, \cdots, n-1$, and we have

$$
\left|\partial_{z_{i}}\left(\partial_{y} z\right)^{n i}\right| \leq C \text { for } z \in Q_{1, \delta^{m-1}} .
$$

Finally, we compute, for $i=1, \cdots, n-1$,

$$
\begin{aligned}
\left|\partial_{z_{n}}\left(\partial_{y} z\right)^{n i}\right| & =\left|\frac{2 \delta\left[\partial_{i} f\left(x_{0}^{\prime}+\delta z^{\prime} / 4\right)-\partial_{i} g\left(x_{0}^{\prime}+\delta z^{\prime} / 4\right)\right]}{\varepsilon+f\left(x_{0}^{\prime}+\delta z^{\prime} / 4\right)-g\left(x_{0}^{\prime}+\delta z^{\prime} / 4\right)}\right| \\
& \leq \frac{C \delta\left|x_{0}^{\prime}+\delta z^{\prime} / 4\right|^{m-1}}{\varepsilon+\left|x_{0}^{\prime}+\delta z^{\prime} / 4\right|^{m}} \leq C \quad \text { for } z \in Q_{1, \delta} .
\end{aligned}
$$

Therefore, (2.21) is verified, and hence (2.20) follows as mentioned above. 
Now we define

$$
S_{l}:=\left\{z \in \mathbb{R}^{n}|| z^{\prime} \mid<1,(l-1) \delta^{m-1}<z_{n}<(l+1) \delta^{m-1}\right\}
$$

for any integer $l$, and

$$
S:=\left\{z \in \mathbb{R}^{n}|| z^{\prime}|<1,| z_{n} \mid<1\right\} .
$$

Note that $Q_{1, \delta^{m-1}}=S_{0}$. As in the proof of Lemma 2.1, we define, for any $l \in \mathbb{Z}$, a new function $\tilde{w}$ by setting

$$
\tilde{w}(z):=w\left(z^{\prime},(-1)^{l}\left(z_{n}-2 l \delta^{m-1}\right)\right), \quad \forall z \in S_{l} .
$$

We also define the corresponding coefficients, for $k=1,2, \cdots, n-1$,

$$
\tilde{b}^{n k}(z)=\tilde{b}^{k n}(z):=(-1)^{l} b^{n k}\left(z^{\prime},(-1)^{l}\left(z_{n}-2 l \delta^{m-1}\right)\right), \quad \forall z \in S_{l},
$$

and for other indices,

$$
\tilde{b}^{i j}(z):=b^{i j}\left(z^{\prime},(-1)^{l}\left(z_{n}-2 l \delta^{m-1}\right)\right), \quad \forall y \in S_{l} .
$$

Then $\tilde{w}$ and $\tilde{b}^{i j}$ are defined in the infinite cylinder $Q_{1, \infty}$. By (2.15), $\tilde{w}$ satisfies the equation

$$
-\partial_{i}\left(\tilde{b}^{i j} \partial_{j} \tilde{w}\right)=0 \text { in } Q_{1, \infty} .
$$

Note that for any $l \in \mathbb{Z}, \tilde{b}(z)$ is orthogonally conjugated to $b\left(z^{\prime},(-1)^{l}\left(z_{n}-2 l \delta^{m-1}\right)\right)$, for $z \in S_{l}$. Hence, by (2.18), we have

$$
\frac{\lambda}{C} \leq \tilde{b}(z) \leq C \Lambda \text { for } z \in Q_{1, \infty}
$$

and, by (2.20),

$$
\|\tilde{b}\|_{C^{\alpha}\left(\bar{S}_{l}\right)} \leq C, \quad \forall l \in \mathbb{Z} .
$$

Apply Lemma 2.1 in [12] on $S$ with $N=1$, we have

$$
\|\nabla \tilde{w}\|_{L^{\infty}\left(\frac{1}{2} S\right)} \leq C\|\tilde{w}\|_{L^{2}(S)}
$$

It follows that

$$
\|\nabla w\|_{L^{\infty}\left(Q_{1 / 2, \delta^{m-1}}\right)} \leq \frac{C}{\delta^{(m-1) / 2}}\|w\|_{L^{2}\left(Q_{1, \delta^{m-1}}\right)} \leq C\|w\|_{L^{\infty}\left(Q_{1, \delta m-1}\right)}
$$

for some positive constant $C$, depending only on $n, \alpha, R_{0}, m, \lambda, \Lambda, \lambda_{1}, \lambda_{2}, \lambda_{3},\|f\|_{C^{2}},\|g\|_{C^{2}}$ and $\|a\|_{C^{\alpha}}$.

By (2.17), we have $\left\|\left(\partial_{z} y\right)\right\|_{L^{\infty}\left(Q_{1, \delta^{m-1}}\right)} \leq C$, where $C$ is independent of $\varepsilon$ and $\delta$. Reversing the change of variables (2.14) and (2.13), we have, by (2.12)

$$
\delta\|\nabla v\|_{L^{\infty}\left(\Omega_{x_{0}, \delta / 8}\right)} \leq C\|v\|_{L^{\infty}\left(\Omega_{x_{0}, \delta / 4}\right)} \leq C\|u\|_{L^{\infty}\left(\Omega_{x_{0}, \delta^{1-\gamma}}\right)} \delta^{\gamma \sigma} .
$$


In particular, this implies

$$
\left|\nabla u\left(x_{0}\right)\right| \leq C\|u\|_{L^{\infty}\left(\Omega_{x_{0}, \delta 1-\gamma}\right)^{-1+\gamma \sigma}} \delta^{-}
$$

and it concludes the proof of Theorem 1.1 for the case $n \geq 3$ after taking $\beta=\gamma \sigma / 2$.

For the case $n=2$, we work with $u$ instead of $v$, and repeat the argument in deriving the first inequality in (2.22), we have

$$
\delta\|\nabla u\|_{L^{\infty}\left(\Omega_{x_{0}, \delta / 8}\right)} \leq C\|u\|_{L^{\infty}\left(\Omega_{x_{0}, \delta / 4}\right)} .
$$

In particular,

$$
\left|\nabla u\left(x_{0}\right)\right| \leq C\|u\|_{L^{\infty}\left(\Omega_{x_{0}, \delta / 4}\right)} \delta^{-1} .
$$

This concludes the proof of Theorem 1.1 for the case $n=2$.

\section{Acknowledgements}

The first author is partially supported by NSF Grants DMS-1501004, DMS-2000261, and Simons Fellows Award 677077. The second author is partially supported by NSF Grants DMS-1501004 and DMS-2000261.

\section{References}

[1] H. Ammari, H. Kang, H. Lee, J. Lee, and M. Lim, Optimal estimates for the electric field in two dimensions, J. Math. Pures Appl., 88(4) (2007), 307-324.

[2] H. Ammari, H. Kang, and M. Lim, Gradient estimates for solutions to the conductivity problem, Math. Ann., 332(2) (2005), 277-286.

[3] I. Babuška, B. Andersson, P. J. Smith, and K. Levin, Damage analysis of fiber composites. I. Statistical analysis on fiber scale, Comput. Methods Appl. Mech. Eng., 172(1-4) (1999), 27-77.

[4] E. Bao, Y. Y. Li, and B. Yin, Gradient estimates for the perfect and insulated conductivity problems with multiple inclusions, Commun. Partial Differential Equations, 35(11) (2010), 1982-2006.

[5] E. Bonnetier and M. Vogelius, An elliptic regularity result for a composite medium with "touching" fibers of circular cross-section, SIAM J. Math. Anal., 31(3) (2000), 651-677.

[6] B. Budiansky and G. F. Carrier, High Shear Stresses in Stiff-Fiber Composites, J. Appl. Mech., 51(4) (1984), 733-735.

[7] H. Dong and H. G. Li, Optimal estimates for the conductivity problem by Green's function method, Arch. Ration. Mech. Anal., 231(3) (2019), 1427-1453.

[8] D. Gilbarg and N. Trudinger, Elliptic Partial Differential Equations of Second Order, Classics in Mathematics, Springer-Verlag, Berlin, 2001.

[9] J. B. Keller, Stresses in narrow regions, J. Appl. Mech., 60(4) (1993), 1054-1056.

[10] Y.Y. Li and L. Nirenberg, Estimates for elliptic systems from composite material, Commun. Pure Appl. Math., 56(7) (2003), 892-925.

[11] Y. Y. Li and M. Vogelius, Gradient estimates for solutions to divergence form elliptic equations with discontinuous coefficients, Arch. Ration. Mech. Anal., 153(2) (2000), 91-151. 
[12] Y. Y. Li and Z. Yang, Gradient estimates of solutions to the insulated conductivity problem in dimension greater than two, arXiv:2012.14056.

[13] X. Markenscoff, Stress amplification in vanishingly small geometries, Comput. Mech., 19(1) (1996), 77-83.

[14] K. Yun, Estimates for electric fields blown up between closely adjacent conductors with arbitrary shape, SIAM J. Appl. Math., 67(3) (2007), 714-730.

[15] K. Yun, Optimal bound on high stresses occurring between stiff fibers with arbitrary shaped cross-sections, J. Math. Anal. Appl., 350(1) (2009), 306-312.

[16] K. Yun, An optimal estimate for electric fields on the shortest line segment between two spherical insulators in three dimensions, J. Differential Equations, 261(1) (2016), 148-188. 